\title{
INSECTOMORPHIC CARGO-CARRYING ROBOT ON A RAFT
}

\author{
YURY F. GOLUBEV and VICTOR V. KORYANOV \\ Keldysh Institute of Applied Mathematics, \\ Russian Academy of Sciences, Moscow, 125047 Russia \\ E-mail: golubev@keldysh.ru,korianov@keldysh.ru
}

\begin{abstract}
The algorithm of the hexapod robot motion control is described. The aim of the motion is to ship the robot on a raft together with a cargo to the other shore of a body of water in the simple case when the robot imparts to the raft an initial push from the shore. The motion consists of transporting the cargo from the shore to the raft, moving the cargo across the moving raft, and carrying the cargo from the raft to the other shore. The algorithm was worked out using computer simulation within the complete dynamical model of motion taking into account the nonstationary action of water on the raft. Computer simulation was performed using the Universal Mechanism software package. Numerical results prove the validity of the algorithm if enough data about the motion for the purpose of control is available.
\end{abstract}

\section{Introduction}

An autonomous mobile robot designed for working on a rough terrain must be able to use environment conditions and various objects available in the area not only as a means for its motion [1] but also as a means for shipping useful cargos [2-4]. A hexapod mobile robot can potentially accomplish such tasks because it is able to release weight from a pair of its legs without violating the static stability [1]. This property is especially important if the robot has no robotic arm or if this arm is out of order [2].

This paper continues the work started in [1]. We assume that the hexapod cannot swim and operate in water; however, it must ship a cargo to the other shore of a narrow body of water. For this purpose, it may use a passive raft [5]. The robot control algorithms are worked out using the mathematical model of the water drag forces described in [6], which is based on the assumption that the effect of the water on raft elements has the "jet" nature. The mathematical model of the raft with the robot and the cargo is described in [7]; it is formed automatically using the Universal Mechanism software [8].

The problem solved in this paper is formulated as follows. Initially, the robot walks on a horizontal plane. There is a long narrow body of water on its path that is perpendicular to the robot's direction of motion. The width of the body of water does not allow the robot to step over it. At the nearest shore, there is a raft on the water. To cross the water obstacle, it is sufficient for the robot to get onto the raft and push off from the shore by its legs. There is a cargo on the shore near the water. The robot should approach the raft, step on it and simultaneously carry the cargo onto it. Then, it should push off from the shore with sufficient strength, float on the raft to the opposite shore, move to the other side of the raft together with the cargo, then step on the opposite shore and carry the cargo onto it. As the robot executes these maneuvers, the raft begins to vibrate relative to the water, and there are impacts due to the cargo transportation [9]. If the robot together with cargo turns out to be outside the raft centerline due to various reasons, then the raft begins to rotate about its vertical axis as the robot walks across the raft and especially as it pushes off the shore. This complicates the problem of control. In this paper, we describe an algorithm for solving the formulated problem. This algorithm was validated using the Universal Mechanism software [8] taking into account the comprehensive dynamics of the system, which 
has 36 degrees of freedom. The computer simulation results show that the proposed robot control algorithm can be implemented in practice.

\section{Crossing the Body of Water by the Robot with a Cargo on a Raft}

The robot design is the same as described in $[1,5]$. The robot has no controllable feet at the ends of its legs, and there are no torques at the points of support [10]. To describe the motion, we use the right-handed absolute reference frame $O \xi \eta \zeta$. The axis $O \eta$ is directed oppositely to the robot motion. The interaction of the raft with the shore is assumed to be viscous-elastic with a considerable predominance of the viscous component [9].

Phase 1. The robot marches to the shore using a "triples" gait in such a way that the cargo is symmetrically positioned relative to the longitudinal symmetry plane of the robot's body. Upon approaching the shore, it changes its gait to "gallop" mode. Then, it transfers its front legs onto the raft and simultaneously moves its body forwards. Next, the rear legs make a step (Fig. 1a). When the sequential transfer of the middle legs is planned, it is checked if they can take the cargo. We assume that the cargo can be taken if $\eta_{c r}<\eta_{i}<\eta_{c f}$, where $\eta_{i}$ are the coordinates of the points of support of the middle legs at the next step and $\eta_{c r}, \eta_{c f}$ are the coordinates of the centers of the vertical cargo faces that are perpendicular to the direction of the robot motion (are parallel to the shores).

Phase 2. If the cargo can be taken, then the target points for the middle legs are chosen on the lateral faces of the cargo. These points are embedded into the cargo to ensure the sufficient pressure and prevent the cargo from slipping out [1]. In addition, the target points are displaced upwards relative to the centers of the lateral faces to prevent the cargo from turning over as it is carried. In order to carry the cargo, additional transfers of the middle legs are planned. The feet of the middle legs are moved to the chosen target points on the lateral faces of the cargo (Fig. 1b); then, the middle legs carry the cargo forwards in the direction of the opposite shore, and after the cargo has been carried, they return to the trace track to the new points of support thus making the next step (Fig. 2a). The duration of each additional carrying over is twice as long as the duration of a regular step cycle. As a result, the middle legs make the next step and simultaneously carry the cargo over at the distance equal to the middle legs step length. The next carrying of the cargo is made when the middle legs are moved in the gallop gait mode. Thus, the robot together with the cargo incrementally moves to the configuration in which the front and middle legs are on the raft, and the body position ensures a sufficient static stability factor for transferring the rear legs, which are still on the shore.

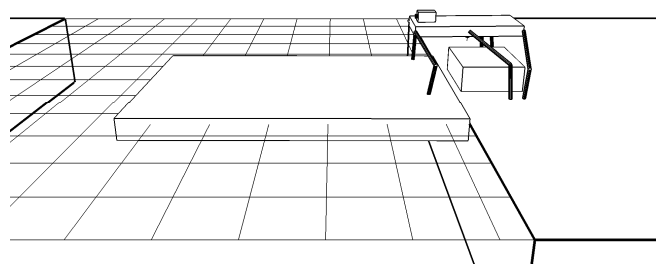

a

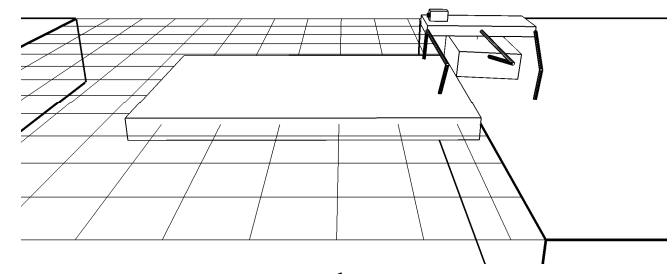

b

Figure 1. Preparing to carry the cargo (a); partial transfer of the cargo onto the raft (b).

Phase 3. Standing on the raft with its front and middle legs, the robot pushes off from the shore, giving the raft and the robot with the cargo an initial velocity $v_{0}$ that is sufficient for reaching the opposite shore (Fig. 2b). After the push, the rear legs are moved to the raft. 


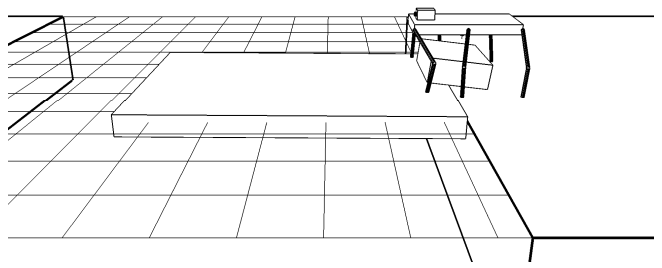

a

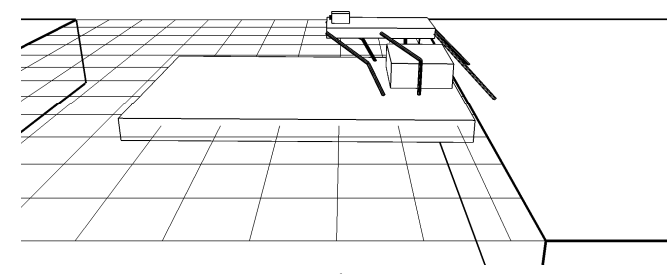

b

Figure 2. The cargo as it is carried over onto the raft (a); pushing off the shore with rear legs (b).

Depending on the distance $S$ between the shores, the velocity $v_{0}$ can be approximately estimated by the formula

$$
v_{0}=v_{\varepsilon} \exp \left[\frac{\kappa_{2}(S-\sigma)}{m+m^{c}+M}\right],
$$

where $v_{\varepsilon}$ is the maximum permissible speed of mooring to the opposite shore, $\sigma$ is the raft's dimension in the direction of the other shore, $M$ is the raft mass, $m$ is the robot mass, $m^{c}$ is the cargo mass, and $\kappa_{2}$ is the coefficient of the water drag taking into account the raft shape. Coefficient $\kappa_{2}$ quadratically depends on the raft speed $[1,6]$.

Phase 4. After the push, the raft floats to the opposite shore. The robot walks on the raft towards the edge with which the raft should moor to the shore; simultaneously, the robot carries the cargo (Fig. 3a). To prevent the robot from falling into water, the leg transfers along with the additional transfers are calculated in the raft's reference frame. The raft's motion on the water is disturbed due to errors in the execution of maneuvers, the weight of the robot with the cargo, and the robot's motion across the raft. During simulation, the robot interacts with the raft only by the forces occurring due to the contact of the robot's feet with the raft's surface. The action of the cargo on the raft is also taken into account via contact forces.

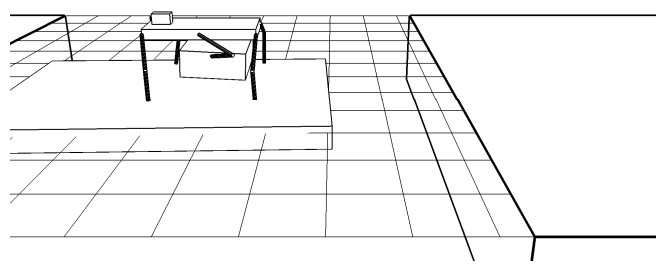

a

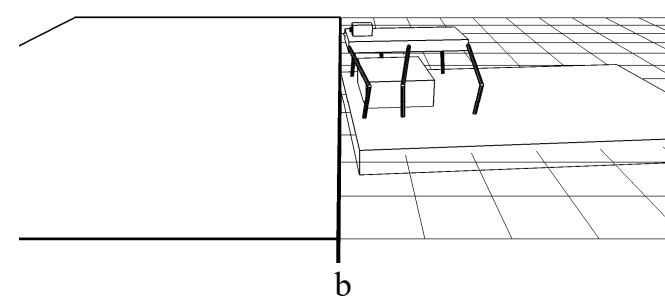

b

Figure 3. Robot with the cargo on the raft (a); mooring (b).

When the cargo meets the raft surface after it is carried over, an impact emerges that affects all elements of the mechanical system and can result in too large deviation of the actual motion from the programmed one. The impact occurs because the raft vibrates on the water and the cargo also vibrates as it is carried over. Moreover, there are errors in the execution of the regular leg transfers. As a result, at the end of the cargo carrying over process, the middle legs with the cargo continue their motion while a point of the cargo has already reached the raft surface, and the time of contact is almost impossible to predict. To attenuate the influence of the inevitable impact, the motion planning algorithm compares the programmed positions of the feet with their actual positions at each integration step. If the mismatch is significant, then the transfer is 
stopped, and the programmed motion is recalculated. If the mismatch is insignificant, then, immediately before the planned contact of the cargo with the raft, the robot body is slightly raised. This motion of the body helps press the supporting feet to the raft surface. After the cargo has contacted the raft surface, the middle legs continue to hold the cargo in a fixed position for a short time thus damping small vibrations of the system relative to the raft.

During the motion, the raft can rotate about its vertical axis because its center was not at the base of the resultant force at the time when the robot pushed off from the shore and because the robot and the cargo continue to move when the raft is floating thus causing additional rotation of the raft about the same axis.

Phase 5. Depending on the width of the body of water, the raft can approach the shore with a certain angle of rotation about its vertical axis (Fig. 3b). The magnitude of this angle can be estimated using the approximate formula

$$
\varphi=\frac{J}{\kappa_{3}} \ln \left\{1+\frac{\kappa_{3}\left(M+m+m^{c}\right)}{J \kappa_{2} h}\left[\exp \left(\frac{\kappa_{2}(S-\sigma)}{m+m^{c}+M}\right)-1\right]\right\},
$$

where $J$ is the effective moment of inertia of the raft and the robot with the cargo about the vertical axis, $\kappa_{3}$ is the water drag factor taking into account the raft shape as it rotates about its vertical axis [6], and $h$ is the distance from the raft's center to the line of action of the total momentum of the forces as the robot pushes off from the shore. If the ratio $\kappa_{3} / J$ is small, formula (1) becomes simpler:

$$
\varphi=\frac{\left(M+m+m^{c}\right)}{\kappa_{2} h}\left[\exp \left(\frac{\kappa_{2}(S-\sigma)}{m+m^{c}+M}\right)-1\right] .
$$

The robot should orient itself in such a way that its front is opposite to the side of the raft that makes the smallest angle with the shore as the raft moors. In particular, if the raft's angle of rotation is less than $\pi / 4$, then the raft will moor to the shore so that its narrow side (opposite to the side that was adjacent to the shore when the raft started its motion) will make the smallest angle with the other shore. Then, the robot will not have to maneuver on the raft when the raft moors. The only maneuver that might be needed is a lateral approach to the corner point of the raft that touches the shore.

Phase 6. To ensure the reliable passage from the raft onto the shore, the raft must be oriented so that its whole side opposite to the robot touches the shore (or at least is not far from the shore). To this end, the robot crouches down in order to reach the shore, transfers its front legs onto the shore, presses its feet to the shore, and pulls the raft in, while turning it in such a way that its whole corresponding side approaches the shore (Fig. 4a).
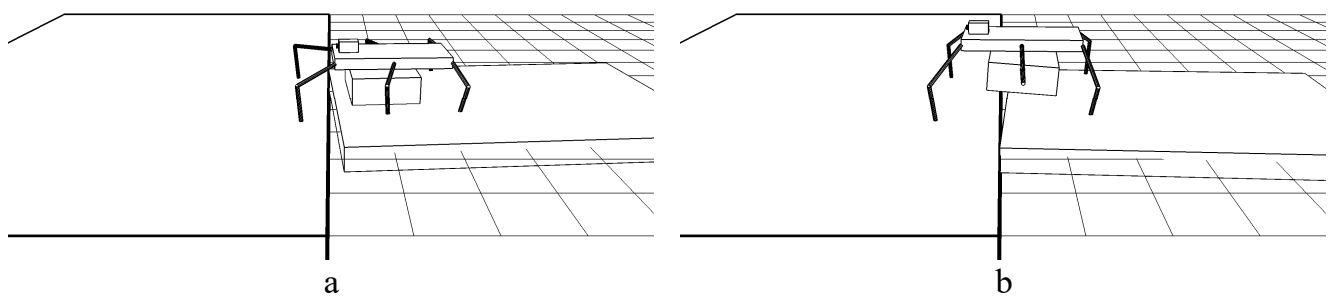

Figure 4. Pulling the raft to the shore (a); carrying the cargo over onto the shore (b). 
The left foot (relative to the way the robot is moving) pulls itself in towards the body while remaining fixed on the shore and the right leg (as well as all of the other legs) remains fixed relative to the body. As this operation is performed, the feet of the front legs can slip relative to the shore due to the considerable horizontal effort, and the corner point of the raft that initially touched the shore can move away from the shore (Fig. 5). If the distance from the shore turns out to be significant, then the robot should continue to pull itself to the shore. Fig. 5 shows the absolute trajectories of the front feet in thin lines.

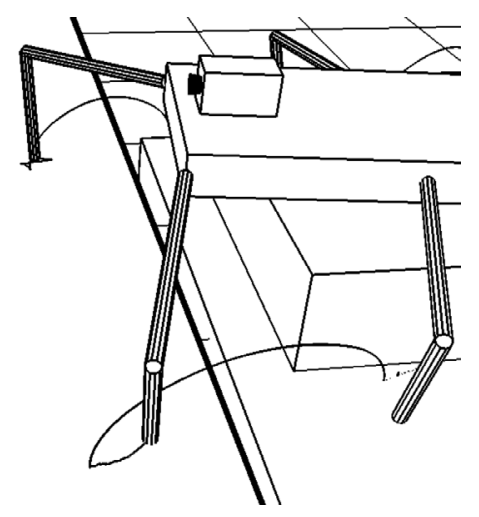

Figure 5. Slippage of the front feet as the raft is pulled to the shore.

When the feet are on the raft, they slightly vibrate due to the disturbed motion of the raft. When the feet are on the shore, the trace of the left foot is monotonic and the trace of the right foot is not monotonic, which reflects the raft's motion relative to the shore when the raft is pulled to the shore. To reduce slippage, the front feet are pressed to the shore with more strength, depending on the displacement of the corresponding foot from its initial point of support. If the foot displacement due to slippage turns out to be unacceptably large (the contact with the shore is lost), then the foot is transferred again to the original point of support, and the pull inward is continued. The raft can also be pulled to the shore using two legs. This procedure is preferable when the load on the legs should be reduced.

Phase 7. As soon as the corresponding side of the raft is sufficiently near the shore, the robot immediately prepares itself for carrying the cargo over. As a result of the front leg slippage relative to the shore in the preceding phase, the robot body can be significantly skewed in the horizontal direction relative to the raft surface. Hence, the robot body can be skewed relative to the cargo. If the cargo is lifted when the robot body is skewed, it is difficult to reliably grip the cargo, and the robot can drop it. To prevent this, the robot's configuration should be corrected before it takes the cargo. For this purpose, it can be required to change the points of support both on the shore (the front legs) and on the raft. The gallop gait is inappropriate for this correction because when a pair of legs is simultaneously transferred in the skewed position, the static stability condition and the reachability of the required points of support can be violated. If the legs are cautiously transferred one by one, then this process can take too much time, and the raft will have time to float off the shore thus making the crossing almost impossible. The point is that the raft is in constant motion, and this can violate the conditions under which the feet can reach possible points of support on the shore. The body correction can be done fairly quickly using the triple gait. In this case, the raft can begin to rock, but the computer simulation shows that this does not violate the overall structure of motion. Making two steps by triples of 
legs, it turns out to be possible to effectively correct the robot position relative to the cargo as required.

After correcting the body position relative to the cargo, the robot lifts its body to walking height and gets onto the shore, while carrying the cargo (Fig. 4b).

\section{Computer Simulation}

Computer simulation was carried out similarly to how it was done in [1, 5]. Individual objects, such as the raft and the robot, interact with the supporting surface and with each other using the friction model based on the viscoelastic interaction of bodies at the contact points. The model of the water drag described in [6] was also used. The motion is controlled by simulating the operation of the electromechanical actuators in the joints. As in reality, the robot motion is controlled by feeding the control voltage to the models of the electromechanical actuators. The ratios of the dimensions of the body, leg links, raft dimensions, the width of the water obstacle, and the dimensions of the cargo are as follows:

$$
\begin{aligned}
& a^{r}: b^{r}: c^{r}: l_{1}: l_{2}: a: b: d: S: a^{c}: b^{c}: d^{c}= \\
& =1: 0.5: 0.1: 0.5: 0.33: 1.5: 1: 0.1: 5.2: 0.3: 0.38: 0.125 .
\end{aligned}
$$

The robot, cargo, and raft masses are related as $16: 10: 840$.

The description of the motion of the robot and other elements of the virtual environment was obtained by numerically solving the differential equations of the complete threedimensional dynamics of the robot along with the other related objects. Since the force interaction was assumed to take place at the points of contact between objects, the mechanical system under consideration has 36 degrees of freedom. The equations of dynamics were synthesized automatically [8].

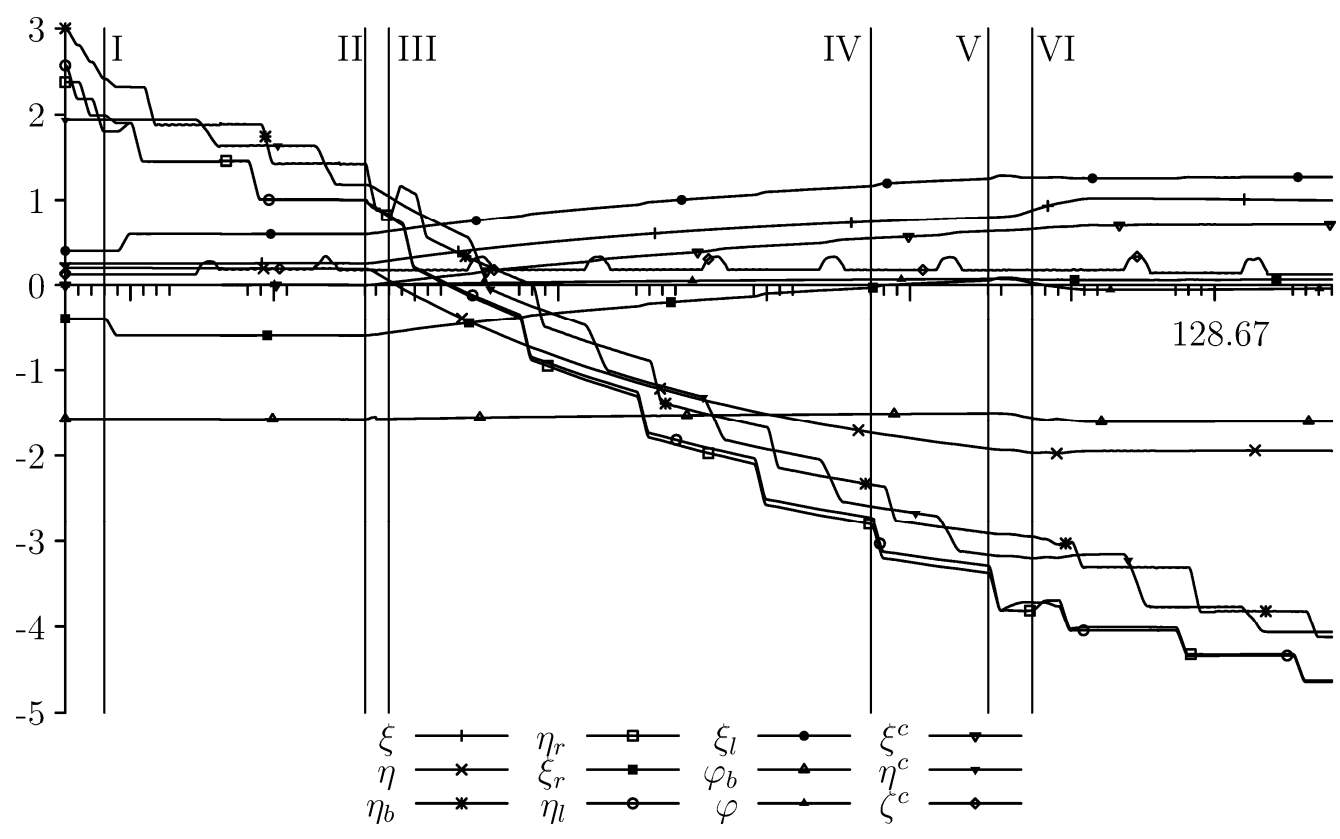

Figure 6. Crossing the body of water by the robot with the cargo on a raft: the dependence of the basic parameters on time. 
Fig. 6 shows the plots of the horizontal coordinates $\xi$ and $\eta$ of the raft's midpoint, where the axis $\eta$ is oriented oppositely to the direction of the robot's motion before it reaches the water; this figure also shows the plots of the body center coordinate $\eta_{b}$, the coordinates $\xi_{r}$ and $\eta_{r}$ of the right front leg, the coordinates $\xi_{l}$ and $\eta_{l}$ of the left front leg, the angle $\varphi_{b}$ of the body rotation about the vertical axis counted from the axis $O \xi$, the angle $\varphi$ of the raft rotation about the vertical axis measured from the axis $O \eta$, and the coordinates $\xi^{c}, \eta^{c}, \zeta^{c}$ of the cargo center. All the coordinates are in meters, and the angles are in radians.

The lines parallel to the $y$-axis denoted by Roman numerals mark the phases of motion.

Line I corresponds to the end of Phase 1 and the beginning of Phase 2. Line II corresponds to the end of Phase 2 and the beginning of Phase 3 , in which the robot pushes off from the shore (along with the raft and the cargo). Line III (the beginning of Phase 4) marks the end of the push and the beginning of the crossing. Between lines III and IV, it can be seen that the raft rotates together with the robot and the cargo. Between lines IV and V, Phase 5 is executed, i.e., the robot carrying the cargo approaches the side of the raft that is the nearest to the opposite shore. Between lines V and VI, Phase 6 is executed, in which the raft is pulled to the shore. Finally, after line VI, the robot with the cargo completes the crossing.

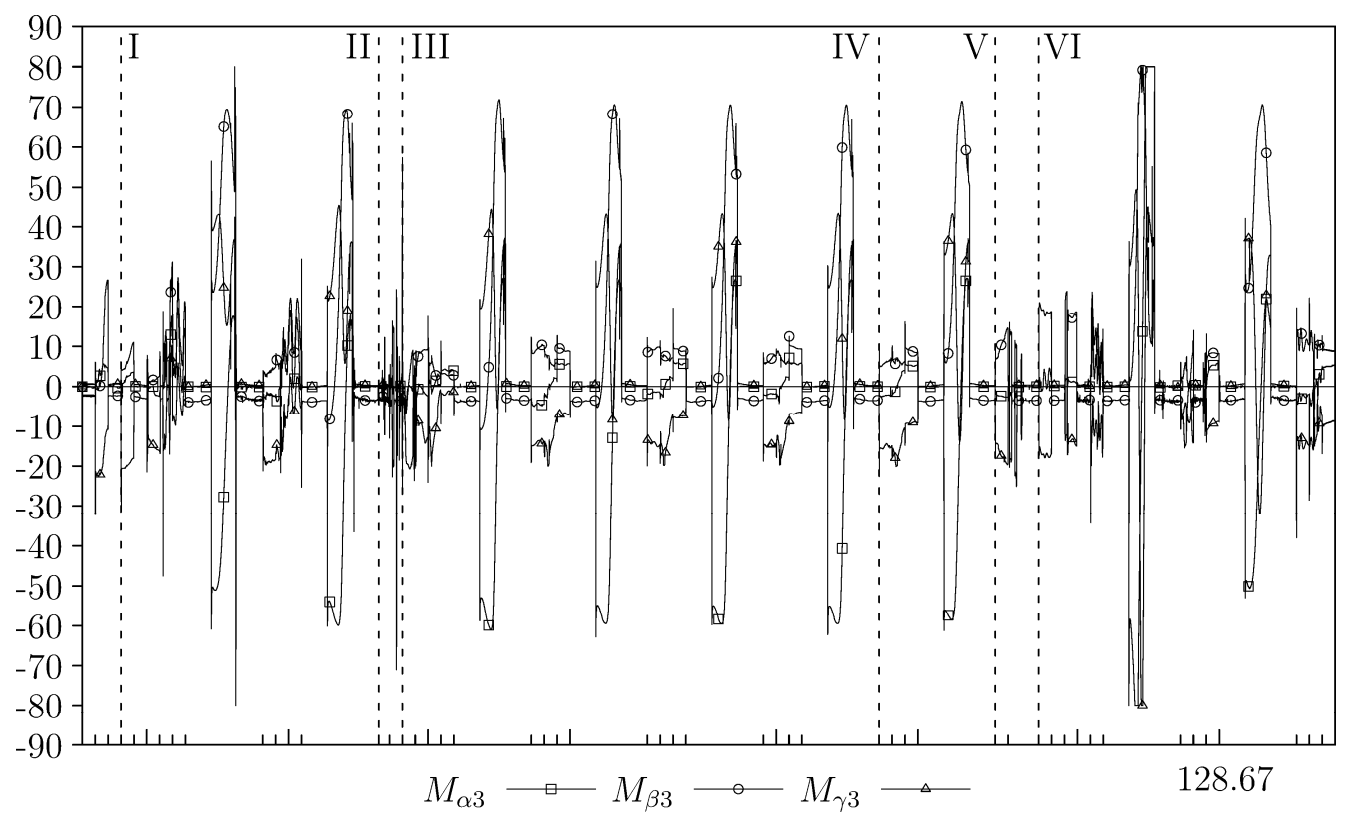

Figure 7. Control torques in joints for right middle leg.

Control torques in joints were limited in absolute value by $80 \mathrm{~N} \cdot \mathrm{m}$. Fig. 7 shows control torques for the right (from the direction of the robot's movement) middle leg number 3 . The construction vertical at the leg attachment point, its hip and shin belong to the same plane. This plane can rotate around the construction vertical by the angle $\alpha_{i} ; \beta_{i}$ is the angle between construction vertical and the hip; $\gamma_{i}$ is the angle between links. The directions of axes from which the angles are counted differs depending on the side of the robot [1].

Impacts on the plots caused by the fact that when the middle legs release the cargo from hold the speed of the feet is non-zero. These impacts did not disrupt the movement in the computer experiments. 


\section{Conclusions}

A new problem of designing the motion of an autonomous insectomorphic robot carrying a cargo that must cross a body of water on a raft is solved. Stable motion of the system as the robot carries the cargo from the shore to the raft, then floats together with the cargo on the raft to the other shore, then moors the raft to the shore, and carries the cargo over to the shore is designed. These maneuvers are complicated by the mobility of the raft on water, possible slippage of the feet as they are put onto the shore when the raft moors, and the risk of mutual leg-crossing.

The proposed algorithms of designing the robot motion for crossing the water obstacle were worked out using computer simulation in a software environment that computes the interactions in a complete 3D dynamic model of the mechanical system consisting of the robot, the cargo, the raft, and the water surface, taking into account the water drag, Coulomb friction and models of the electromechanical actuators in the robot joints.

It is shown that the programmed motion which is calculated during the maneuvering ensuring that the system as a whole moves as required can be implemented if the friction coefficient does not exceed 1. In the present paper, this value of the coefficient of the static friction of the foot on the supporting surface is considered to be limiting.

The stabilization of the robot's motion in the neighborhood of the designed programmed motion is based on the piecewise feedback formed discretely with the step equal to the integration step. The feedback is computed based on the mismatch between the actual and the prescribed joint angles so as to minimize the angular velocities. The transient processes in the joint angles are not destructive to the designed motion of the robot's body in all phases of the system's motion.

\section{Acknowledgments}

This work was supported by the Russian Foundation for Basic Research, project no. 19-0100123.

\section{References}

1. Yu. F. Golubev and V. V. Koryanov, Extreme Locomotion Capabilities of Insectomorphic Robots (Keldysh Inst. Appl. Math., Moscow, 2018) [in Russian].

2. Synergy of Automation, IoT and AI. CLAWAR 2019 Proceedings. CLAWAR Association Ltd, UK. ISBN: 978-1-9164490-2-2.

3. www.bostondynamics.com/bigdog.

4. Gita (mobile carrier). https://en.wikipedia.org/wiki/Gita_(mobile_carrier)

5. Yu. F. Golubev and V. V. Koryanov, Shipping Cargo on a Raft by an Insectomorphic Robot, J. Comput. Syst. Sci. Int. Vol. 57, No. 5, pp. 813-821 (2018).

6. Yu. F. Golubev, Non-stationary model of forces for water influence on a rectangular raft, KIAM Preprint No. 53 (Keldysh Inst. Appl. Math., Moscow, 2016). DOI: 10.20948/prepr2016-53 [in Russian]

7. Yu. F. Golubev, Non-stationary model of perturbed dynamics of a rectangular raft on a calm water, KIAM Preprint No. 54 (Keldysh Inst. Appl. Math., Moscow, 2016). DOI: 10.20948/prepr-2016-54 [in Russian]

8. Universal Mechanism. Modeling the Dynamics of Mechanical Systems, http://www.umlab.ru.

9. H. R. Hertz, The Principles of Mechanics Presented in a New Form (Dover, New York, 2004).

10. D. E. Okhotsimskii and Yu. F. Golubev, Mechanics and Motion Control of Automatic Legged Vehicle (Nauka, Moscow, 1984) [in Russian]. 